\title{
Polyunsaturated fatty acids modify colorectal epithelial cell cytokine expression in a leucocyte co-culture model in response to probiotic bacteria
}

\author{
K. L. Bentley-Hewitt ${ }^{1}$, C.-E. De Guzman ${ }^{2}$, J. Sutherland ${ }^{2}$, T. Mandimika ${ }^{2}$, A. Narbad ${ }^{1}$ and E. K. Lund ${ }^{1}$ \\ ${ }^{1}$ Institute of Food Research, Norwich, UK and ${ }^{2}$ New Zealand Plant and Food Research, Palmerston North, New Zealand
}

Diets high in $n-3$ polyunsaturated fatty acids (PUFAs) have been linked to improved colorectal health ${ }^{(1)}$. One possible mechanism underlying this observation is that different PUFAs affect the immune response of epithelial cells to beneficial bacteria such as probiotics. The aim of this study was therefore to test whether pre-incubation of cells with different PUFAs would modify the epithelial cell response to probiotic bacteria.

We employed a co-culture system as described previously ${ }^{(2)}$ in which three different colorectal cell lines (Caco-2, HT-29 and the mucin producing line HT29-MTX) were grown to confluence on semi-permeable membranes in a six-well plate and then treated with different PUFAs (arachidonic acid C20:4 n-6, AA; eicosapentaenoic acid C20:5 n-3, EPA and docosahexaenoic acid C22:6 n-3, DHA) for 12 h with human peripheral blood mononuclear cells (PBMC) in the basolateral compartment. The apical medium was then removed and cells washed 3 times before adding the probiotic bacteria L. gasseri $\left(1 \times 10^{9}\right.$ per well) to the apical compartment in $1 \mathrm{ml}$ RPMI + glutamax for $3 \mathrm{~h}$. Unattached bacteria were removed and cells left for a further $6 \mathrm{~h}$ in medium. Media from both compartments were collected for measurement of cytokines by ELISA and cells removed into RNA extraction buffer. After an initial screening to assess which cytokines are expressed in the co-culture system, gene expression of IL-8, HSP25, HSP72 and TGF- $\beta$ was measured by quantitative RT-PCR using a Lightcycler ${ }^{\circledR} 480$ system, Roche with probes selected from the universal library.

A complex picture emerged from these studies. Generally the response to L. gasseri was different in Caco-2 cells than that in either form of HT29 cell (Table 1).

\begin{tabular}{|c|c|c|c|c|}
\hline & TGF- $\beta$ & IL-8 & HSP72 & HSP25 \\
\hline HT29 & $0.08(0.02)^{* *}$ & $1.02(0.21)^{* *}$ & $0.37(0.83)$ & $0.04(0.06)$ \\
\hline HT29-MTX & $2.48(1.78)^{* * *}$ & $3.89(1.53)^{* *}$ & $0.76(2.56)$ & $0.51(0.48)$ \\
\hline Caco-2 & $0.26(0.78)$ & $5.12(3.35)$ & $1.72(0.84)^{* *}$ & $3.04(0.52)^{* *}$ \\
\hline
\end{tabular}

In the absence of $L$. gasseri all PUFAs increased expression of TGF- $\beta$ in HT29 cells, while only EPA had this effect in MTX cells. In Caco-2 cells all PUFAs down-regulated this gene. AA increased HSP72 expression in Caco-2 cells. Generally, the bacterial response tended to dominate the effects of PUFAs on L. gasseri treated cells, but we did observe an interaction between bacterial and PUFA exposure in HT29-MTX cells in relation to HSP72 and TGF- $\beta$. In the latter case, AA reduced expression, while EPA increased it.

In conclusion, we can say that there are interactions between PUFAs and bacterial exposure. In the case of TGF- $\beta$ this is of particular interest as we have found differential effects of $n-3$ and $n-6$ fatty acids and this protein is known to be involved in induction of tolerance ${ }^{\left({ }^{3}\right)}$.

1. Lund E (2009) In Chemoprevention of Cancer and DNA Damage by Dietary Factors (S Knasmüller, D DeMarini, IT Johnson et al., editors). pp 749760. Oxford: Wiley-Blackwell (ISBN: 978-3-527-32058-5).

2. Haller D, Holt L, Parlesak A et al. (2004) Immunology 112, 310-320.

3. Takebayashi K, Hokari R, Kurihara C et al. (2009) Microcirculation 16, 251-264. 